\title{
Avaliação clínica de reabsorção radicular externa em dentes desvitalizados submetidos ao clareamento
}

\section{Clinical evaluation of external radicular resorption in non-vital teeth submitted to bleaching}

\author{
Alessandro Dourado Loguercio* \\ Daniela Souza** \\ Adriana Soares Floor** \\ Mauro Mesko** \\ Alcebiades Nunes Barbosa*** \\ Adair Luiz Stefanello Busato****
}

\begin{abstract}
RESUMO: O propósito deste estudo foi avaliar a presença de reabsorção cervical externa em pacientes submetidos ao clareamento de dentes desvitalizados. Os pacientes avaliados tiveram pelo menos um dente desvitalizado clareado entre os anos de 1986 a 1996. Os pacientes foram submetidos à técnica de clareamento com perborato de sódio e peróxido de hidrogênio, de acordo com a técnica descrita por Busato et al. ${ }^{5,6}$. Dos 193 pacientes chamados para que os dentes clareados fossem examinados clínica e radiograficamente, apenas 43 pacientes compareceram (54 dentes) com uma média de tempo após o clareamento de 3,5 anos. Os resultados permitiram concluir que em nenhum dos dentes examinados foi possivel observar indícios de reabsorção cervical externa.

UNITERMOS: Reabsorção da raiz; Clareamento de dente; Peróxido de hidrogênio.
\end{abstract}

ABSTRACT: The purpose of this study was to evaluate the presence of external resorption in non-vital teeth submitted to bleaching. The evaluated patients had at least one non-vital tooth, which had been bleached between 1986 and 1996. All teeth were submitted to bleaching with hydrogen peroxide and sodium perborate, as described by Busato et $a$. $^{5,6}$. From 193 patients recalled for clinical and radiographic evaluation of bleached teeth, only 43 attended (54 teeth). The average time elapsed after bleaching was 3.5 years. The results revealed that none of the examined teeth had any degree of external cervical resorption.

UNITERMS: Root resorption; Tooth bleaching; Hydrogen peroxide.

\section{INTRODUÇÃO}

O escurecimento dos dentes é motivo de grande preocupação por parte dos pacientes. Nesse contexto, o clareamento de dentes desvitalizados é uma terapia muito requisitada na prática diária do consultório ${ }^{7}$.

A etiologia do escurecimento de dentes desvitalizados é bem conhecida. Essa mudança cromática pode ser ocasionada por uma hemorragia advinda de trauma, técnica terapêutica inadequada, espaço de tempo entre o traumatismo e o atendimento odontológico e tempo de permanência da restauração provisória, após o tratamento de canal' ${ }^{2,6}$.

Independentemente desses fatores causais, todo profissional deve estar bem preparado para diagnosticar corretamente a causa da alteração de cor, pois essa é uma condição indispensável para o sucesso do procedimento.

A técnica de clareamento é um procedimento utilizado há muito tempo, e tem três vantagens indiscutiveis, como: evitar o desgaste de estrutura dentária em comparação com outros procedimentos, obter resultados estéticos satisfatórios comprovados em longo prazo e onerar menos o paciente ${ }^{6}$.

Contudo, sabe-se também que há efeitos deletérios para os dentes e as estruturas de suporte ${ }^{9}$, sendo a reabsorção cervical externa (RCE) a mais grave descrita na literatura.

Desde o relato de Harrington, Natkin ${ }^{14}$ (1979), outras publicações têm sido realizadas buscando associar o clareamento de dentes aos problemas de RCE. Apesar desta associação não estar com-

\footnotetext{
*Doutorando em Materiais Dentários da Faculdade de Odontologia da Universidade de São Paulo.

${ }^{* *}$ Cirurgiões-dentistas.

***Professor Adjunto; ****Professor Titular da Faculdade de Odontologia da Universidade Luterana do Brasil.
} 
Loguercio AD, Souza D, Floor AS, Mesko M, Barbosa AN, Busato ALS. Avaliação clínica de reabsorção radicular externa em dentes desvitalizados submetidos ao clareamento. Pesqui Odontol Bras 2002;16(2):131-135.

pletamente elucidada, já foi demonstrado em animais (cães) que agentes clareadores (peróxido de hidrogênio) induzem a ocorrência de $\mathrm{RCE}^{24}$.

Dessa forma, especula-se que a associação entre o emprego do peróxido de hidrogênio e o aparecimento da RCE é causada por alguns fatores. O peróxido de hidrogênio em altas concentrações tem um baixo $\mathrm{pH}$, que poderá ocasionar desnaturação protéica (orgânica) e desmineralização (inorgânica) que levará a um aumento da permeabilidade dentinária na região, fazendo com que os gases da reação química do perborato de sódio com o peróxido de hidrogênio (técnica mediata) ou do peróxido de hidrogênio com calor (técnica imediata) possam chegar até a superfície externa da raiz ${ }^{18,19,26}$.

Isso é agravado, segundo Rotstein et al. ${ }^{26}$ (1991) por falhas na junção amelocementária que propiciam uma margem de periodonto exposto. Além disso, a associação com calor (técnica imediata) leva a um aumento da reatividade do peróxido de hidrogênio, mas também a um aumento da permeabilidade dentinária devido ao coeficiente de expansão térmico linear da dentina e um aumento das trincas, notadamente nos casos de dentes traumatizados.

Essas reações podem determinar uma agressão ao periodonto, que responderá por meio de uma resposta auto-imune (inflamatória), enviando osteoclastos para eliminarem o corpo estranho e isso levará a uma reação localizada de reabsorção externa ${ }^{20,24}$.

Sendo assim, neste estudo procurou-se, por meio da avaliação clínica e radiográfica observacional retrospectiva, detectar a presença de $\mathrm{RCE}$ em dentes submetidos a uma técnica de clareamento de dentes desvitalizados em que foi associado o perborato de sódio com peróxido de hidrogênio no período de 1986 a 1996.

\section{MATERIAL E MÉTODOS Realização do clareamento}

Foi realizada uma avaliação clínica e radiográfica prévia para que fosse indicado o tratamento clareador. Ao exame clínico, observava-se a integridade do remanescente dental, seu grau de escurecimento e as suas condições periodontais. Ao exame radiográfico, foram observadas as condições endodônticas (vedamento no periápice) e a situação periodontal (óssea).

Depois de definida a indicação, a técnica empregada no clareamento foi a de Busato et al. ${ }^{5,6}$ (1986, 1997), descrita a seguir: 1) foi registrada a cor inicial com auxílio de uma escala de cores; 2) foi realizada a profilaxia, a proteção dos tecidos moles com vaselina cremosa e o isolamento absoluto (preferencialmente apenas do dente a ser clareado); 3) foi executada a remoção da restauração provisória e/ou cárie abordando corretamente o dente a ser clareado com instrumentos rotatórios e/ou manuais. Logo a seguir, 4) foi iniciada a desobstrução da entrada do canal (2-3 $\mathrm{mm}$ além da junção amelocementária) com instrumentos rotatórios e/ou manuais aquecidos. Nessa região, 5) foi realizado o vedamento cervical, em geral, com cimento de silicato no início e depois com o cimento de ionômero de vidro ${ }^{5,6}$.

Numa segunda sessão, foi realizada a técnica termocatalítica (imediata). A técnica consiste em levar um instrumento aquecido sobre um chumaço de algodão umedecido em peróxido de hidrogênio a $35 \%$, que estava posicionado na superficie vestibular do dente. Este processo era realizado por no máximo três vezes, com intervalos de três a cinco minutos entre as aplicações, até que o algodão ficasse completamente seco. Na mesma sessão utilizava-se a técnica de clareamento intracoronária (mediata). Uma mistura de peróxido de hidrogênio a 35\% associada ao perborato de sódio, na forma de uma pasta cremosa e brilhante era colocada na câmara pulpar em contato com a face vestibular. Sobre essa pasta era adaptado um algodão e uma restauração provisória (óxido de zinco e eugenol modificado). Essa sessão era realizada uma semana após a primeira, quando se verificava a melhora ou não da cor. Nas sessões subseqüentes (no máximo de 5), apenas realizava-se a técnica mediata. Após o dente alcançar a cor desejada, ele era limpo e restaurado com sistema adesivo e resina composta e/ou cimento ionomérico, de acordo com cada caso.

\section{Realização do exame de retorno dos pacientes}

Todos os pacientes (193) que foram submetidos a essa técnica no período de 1986 a 1996 foram chamados para nova avaliação clínica e radiográfica dos dentes clareados e preenchimento de uma ficha com dados sobre o tratamento.

\section{RESULTADOS}

Dos 193 pacientes, apenas 43 compareceram às avaliações, num total de 54 dentes tratados endodonticamente e que foram submetidos a tratamento clareador. O tempo após o clareamento variava de 1 a 10 anos, com uma média de tempo em torno de 3,5 anos. Destes, quatro dentes haviam 
Loguercio AD, Souza D, Floor AS, Mesko M, Barbosa AN, Busato ALS. Avaliação clínica de reabsorção radicular externa em dentes desvitalizados submetidos ao clareamento. Pesqui Odontol Bras 2002;16(2):131-135.

fraturado após o clareamento e um dente fraturou durante o clareamento. Esses cinco dentes foram excluídos das outras avaliações (Figura 1).

No questionário, algumas perguntas foram destacadas para ilustrar detalhes importantes dentro da técnica de clareamento. Foi perguntado: qual a causa do tratamento endodôntico? $77 \%$ responderam (38 dentes) que a causa era a cárie; 10,5\% que era devido ao trauma e em 7 casos não sabiam descrever a causa. Também foi questionado ao paciente se o escurecimento ocorreu antes ou após a endodontia: 14,3\% (7) não sabiam, 36,7\% (18) responderam que o escurecimento já existia antes do tratamento de canal e 49\% (24) disseram que o escurecimento ocorreu pós-endodontia.

Ao exame clínico e radiográfico de controle, não foi possivel detectar nenhum dente com possivel suspeita de reabsorção cervical externa (Figura 2). Não se conseguiu estabelecer uma correlação do percentual de recidiva do escurecimento, entretanto em alguns casos (Figura 3) a recidiva do procedimento clareador foi visivel.

\section{DISCUSSÃO}

Mesmo que em nenhum dos casos tenham sido encontradas $\mathrm{RCE}$, é interessante discutir alguns tópicos da técnica de clareamento.

Segundo Macisaac, Hoen ${ }^{20}$ (1994), os relatos de $\mathrm{RCE}$ associados ao processo de clareamento publicados na literatura têm em comum alguns fatores: em $100 \%$ dos casos, o vedamento cervical era inexistente e, em 84\% usou-se a técnica termocatalí-

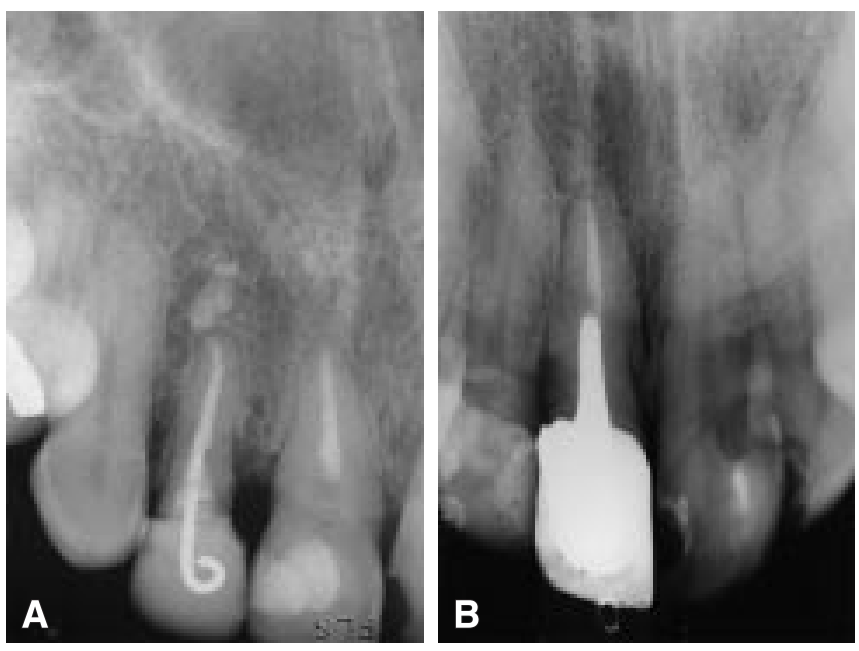

FIGURA 1 - A e B: radiografias periapicais de incisivos centrais direitos que receberam o tratamento clareador (1991 e 1989) e que se apresentaram reconstruídos na reavaliação feita em 1996. Os pacientes relataram que a fratura ocorreu após o clareamento. tica. Além desses, o clareamento foi realizado em $80 \%$ dos casos na mesma sessão da obturação do canal radicular e em $74 \%$ o trauma foi o fator etiológico a desencadear a necessidade de endodontia a que o dente havia sido submetido.

Desde a indicação por Lado et al. ${ }^{18}$ (1983) da colocação de um material para vedar a região cervical, todos os autores concordam que esta é a melhor forma de prevenir que os agentes clareadores possam atingir via região cervical ou via canal radicular o periodonto. Entretanto, existe uma enorme controvérsia com relação ao material a ser utilizado para o vedamento cervical e é preciso que mais estudos sejam realizados para verificação do melhor material a ser utilizado nessa região, ${ }^{5,8,25}$.

A opção, na época, pelo ionômero de vidro foi devido as suas propriedades de adesão a estrutura

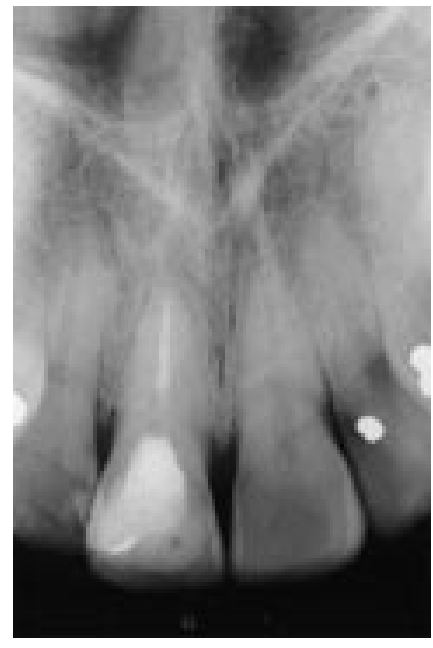

FIGURA 2 - Radiografia periapical de dente submetido ao tratamento clareador em 1990 e radiografado em $1996 \mathrm{sem}$ suspeita de reabsorção cervical.

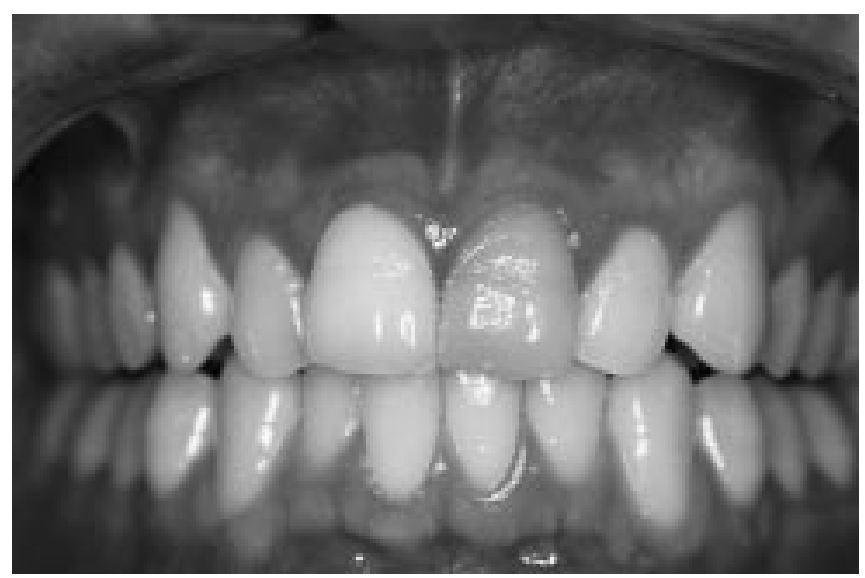

FIGURA 3 - Dente 21 que sofreu clareamento em 1987 e foi examinado em 1996. Observe a descoloração. Segundo o paciente, o escurecimento não era tão intenso como da primeira vez, e a reincidência havia acontecido havia aproximadamente 2 anos. 
Loguercio AD, Souza D, Floor AS, Mesko M, Barbosa AN, Busato ALS. Avaliação clínica de reabsorção radicular externa em dentes desvitalizados submetidos ao clareamento. Pesqui Odontol Bras 2002;16(2):131-135.

dentária, biocompatibilidade e coeficiente térmico linear muito próximo ao dos tecidos duros do dente. Parece que o mais importante não é o material restaurador a ser utilizado, mas sim o cuidado de que o procedimento de clareamento seja realizado ao redor de uma semana após a obturação definitiva do $\mathrm{canal}^{7}$.

Deperalta et al. ${ }^{10}$ (1991) compararam a utilização ou não do vedamento cervical em relação ao clareamento imediato ou uma semana após a obturação do canal. Demonstraram que o fator mais importante foi a espera por 7 dias, independentemente da utilização ou não do material para vedamento. Isso justificaria, em grande parte, os bons resultados obtidos com o ionômero de vidro convencional utilizado.

É importante que o material para vedamento cervical seja colocado imediatamente após ter sido realizado o tratamento endodôntico, haja vista que isso permite a completa reação de presa do material até a próxima sessão, mas principalmente evita que o dente não escurecido possa ficar pigmentado (demora a ser restaurado definitivamente), principalmente em se tratando de restaurações provisórias que tenham eugenol.

Outro fato interessante a ser comentado é a fratura ocorrida em alguns dentes. A relação entre clareamento e enfraquecimento do dente foi demonstrada inicialmente por alguns autores ${ }^{11}$, mas foi contestada por outros estudos ${ }^{4,22}$. Isto indicaria que o dente clareado não fica mais fraco após o clareamento. Durante o clareamento de dentes desvitalizados, a câmara pulpar é vedada com um material que não devolve a resistência ao elemento dental, sendo isso agravado quando já houve envolvimento das arestas marginais que são consideradas regiões de reforço em dentes anteriores ${ }^{4}$. Portanto, o procedimento deve ser realizado com o maior número de cuidados para evitar maiores danos ao remanescente coronário.

Sempre que um dente a ser clareado tiver sofrido trauma, a primeira opção deve ser o uso de uma técnica que utilize substâncias menos cáusticas (peróxido de hidrogênio em baixas concentrações, ou mesmo a água, associando-se ao perborato de sódio), em detrimento da utilização do peróxido de hidrogênio a 35\% associado ao calor (técnica termocatalítica). Esta associação (trauma e substância clareadora) não traz grandes prejuízos ao elemento dental, já que a queda de pH, com substâncias menos cáusticas, é muito pequena ${ }^{23,27}$. Outro fator a ser considerado é que o peróxido de hidrogênio é uma substância extremamente instável (6 meses), e isso sem dúvida contribuiu para que uma substância menos agressiva estivesse sendo utilizada ${ }^{13,16}$.

Além do mais, estudos demonstraram a efetividade de técnicas com substâncias menos cáusticas no clareamento de dentes. Apenas houve diferença no tempo de clareamento ${ }^{16,26,27}$.

A ausência de casos de reabsorção neste estudo se deve acima de tudo à utilização do vedamento cervical, baixo número de dentes traumatizados e de se ter iniciado o clareamento apenas uma semana após a obturação do canal, conforme indica Macisaac, Hoen ${ }^{20}$ (1994).

Estes resultados vão ao encontro de outras pesquisas clínicas. Em estudos em que foi utilizado o vedamento cervical ${ }^{1,3,21}$, ou a associação de substâncias menos caústicas, como o perborato de sódio mais água ${ }^{17}$, não foi encontrado nenhum caso de RCE.

Em contrapartida, em estudos em que alguns desses fatores previamente $\operatorname{citados}^{20}$ foram negligenciados, sempre houve casos de reabsorção. No estudo de Friedman et al. ${ }^{12}$ (1988), foram encontrados 4 casos de RCE em 58 casos. Em nenhum dente, foi utilizado o vedamento cervical. Em outra pesquisa $^{15}$, também foram encontrados 4 casos de RCE com tempo de 1-19 anos de acompanhamento, sendo que nesse estudo o vedamento cervical foi realizado com guta-percha (material não adesivo).

O clareamento de dentes sem vitalidade seja pelo seu alto índice de sucesso, seja por ser uma técnica extremamente conservadora e de baixo custo, é um procedimento com grande indicação, contudo alguns detalhes devem ser atentados para que problemas como o de reabsorção não venham a comprometer o sucesso clínico do tratamento. Mais estudos devem ser realizados para comprovar os resultados demonstrados neste estudo.

\section{CONCLUSÃO}

Com base nos dados encontrados, é possivel concluir que não ocorreu nenhum caso de reabsorção cervical externa nos 54 dentes estudados com um tempo médio de 3,5 anos com a técnica de clareamento de dentes desvitalizados preconizada.

\section{AGRADECIMENTOS}

Gostariamos de agradecer a Paulo Cesar Roman (in memorian) pelo exemplo de amor a vida, ao Prof. Dr. Flávio Fernando Demarco pela sugestão do estudo, aos funcionários do serviço de triagem da Faculdade de Odontologia de Pelotas - RS (UFPEL) e o apoio parcial da FAPESP 99/05124-0. 
Loguercio AD, Souza D, Floor AS, Mesko M, Barbosa AN, Busato ALS. Avaliação clínica de reabsorção radicular externa em dentes desvitalizados submetidos ao clareamento. Pesqui Odontol Bras 2002;16(2):131-135.

\section{REFERÊNCIAS}

1. Abbott PV. Internal bleaching of teeth. An analysis of 203 cases [abstract 14] J Dent Res 1995;74:747.

2. Baratieri LN, Monteiro Junior S, Andrada MAC, Vieira LCC. Clareamento Dental. São Paulo: Quintessence Books; 1993.

3. Beviláqua MV, Fernandes $\mathrm{ACD}$, Gurgel $\mathrm{ED}$, et al. Reabsorção cervical externa após clareamento dental. RGO 1995;43:81-4.

4. Bezerra RB, Navarro MFL, Souza Junior MHS, et al. Influência das cristas marginais na resistência à fratura de dentes despolpados submetidos ao tratamento clareador. Rev Odontol Univ São Paulo 1993;7:125-9.

5. Busato ALS, Macedo RP, Panitz P, et al. Clareamento de dentes escurecidos. RGO 1986;34:497-500.

6. Busato ALS, Barbosa AN, Baldissera RA, Bueno MP. Dentística: restaurações em dentes anteriores. São Paulo: Artes Médicas; 1997.

7. Brighton DM, Harrington GW, Nicholls JI. Intracanal isolating barriers as they relate to bleaching. J Endod 1994;20:228-32.

8. Costas FL, Wong M. Intracoronal isolating barriers: effect of location on root leakage and effectiveness of bleaching agents. J Endod 1991;17:365-8.

9. Demarco FF, Garone Netto N. Efeitos adversos do clareamento em dentes endodonticamente tratados. Rev Odontol Univ São Paulo 1995;9:51-8.

10. Deperalta AA, Joyner HN, Burgess JO, et al. Apical leakage of bleaching agents through an intermediate dental base material. Gen Dent 1991;39:448-50.

11. Francischone CE, Francisconi PAS, Souza Junior MHS, et al. Resistência à fratura de dentes despolpados tratados com agentes clareadores. Rev Bras Odontol 1990;43:2-5.

12. Friedman $\mathrm{S}$, Rotstein I, Libfeld $\mathrm{H}$, et al. Incidence of external root resorption and esthetic results in 58 bleached pulpless teeth. Endod Dent Traumatol 1988;4:23-6.

13. Hardman PK, Moore DL, Petteway GH. Stability of hydrogen peroxide as a bleaching agent. Gen Dent 1985; 33:121-2.
14. Harrington G, Natkin E. External resorption associated with bleaching of pulpless teeth. J Endod 1979;5:344-8.

15. Heithesay GS, Dhalstrom SW, Marin PD. Incidence of invasive cervical resorption in bleached root-filled teeth. Aust Dent J 1994;39:82-7.

16. Ho S, Goering AC. An in vitro comparison of different bleaching agents in the discolored tooth. J Endod 1989; 15:106-11.

17. Holmstrup G, Palm AM, Lambjerg-Hansen H. Bleaching of discoloured root-filled teeth. Endod Dent Traumatol 1988;4:197-201.

18. Lado EA, Stanley HR, Weinsman MT. Cervical resorption in bleached teeth. Oral Surg Oral Med Oral Pathol 1983; 55:78-80.

19. Lewinstein I, Hirschfeld Z, Stabholz A, et al. Effect of hydrogen peroxide and sodium perborate on the microhardness of human enamel and dentin. J Endod 1994;20:61-3.

20. Macisaac AM, Hoen MM. Intracoronal bleaching: concerns and considerations. J Can Dent Assoc 1994;60:57-64.

21. Monnerat AF, Dias K, Filho HA. Clareamento intracoronário pela técnica "walking bleach": um estudo de 2 anos. Rev Bras Odontol 1995;53:53-5.

22. Moraes SH, Aragão EM, Heck AR, et al. Resistência à fratura de dentes despolpados e clareados. RGO 1991;39:309-10.

23. Rotstein I, Friedman S. PH variation among materials used for intracoronal bleaching. J Endod 1991;17:376-9.

24. Rotstein I, Friedman S, Mor C, et al. Histological characterization of bleaching-induced external root resorption in dogs. J Endod 1991;17:436-41.

25. Rotstein I, Lehr Z, Gedalia I. Effect of different protective base materials on hydrogen peroxide leakage during intracoronal bleaching in vitro. J Endod 1992;18:114-7.

26. Rotstein I, Torek Y, Lewinstein I. Effect of bleaching time and temperature on the radicular penetration of hydrogen peroxide. Endod Dent Traumatol 1991;7:196-8.

27. Weiger R, Kuhn A, Löst C. Effect of various types of sodium perborate on the $\mathrm{pH}$ of bleaching agents. J Endod 1993; 19:239-41.

Recebido para publicação em 01/11/2000

Enviado para reformulação em 01/10/2001

Aceito para publicação em 10/12/2001 